\title{
A Methodology to Estimate Capacity Impact due to Connected Vehicle Technology
}

\author{
Daiheng Ni, Jia Li, Steven Andrews, and Haizhong Wang \\ Department of Civil and Environmental Engineering, University of Massachusetts Amherst, Amherst, MA 01003, USA \\ Correspondence should be addressed to Daiheng Ni, ni@ecs.umass.edu
}

Received 16 March 2011; Revised 21 June 2011; Accepted 7 September 2011

Academic Editor: Nandana Rajatheva

Copyright () 2012 Daiheng Ni et al. This is an open access article distributed under the Creative Commons Attribution License, which permits unrestricted use, distribution, and reproduction in any medium, provided the original work is properly cited.

\begin{abstract}
Recent development in connected vehicle technology or equivalently vehicular ad hoc networks (VANET) has stimulated tremendous interests among decision makers, practitioners, and researchers due to the potential safety and mobility benefits provided by these technologies. A primary concern regarding the deployment of connected vehicle technology is the degree of market penetration required for effectiveness. This paper proposes a methodology to analyze the benefit of highway capacity gained from connected vehicle technology. To fulfill this purpose, a model incorporating the effects of connected vehicle technology on car following is formulated, building on which a rough estimate of the resulting capacity gain is derived. A simulation study is conducted to verify the model, and an illustrative example is provided to show the application of the methodology. This work provides decision makers and practitioners with a basic tool to understand the mobility benefit obtained from connected vehicle technology and how such benefit varies as market penetration changes.
\end{abstract}

\section{Introduction}

Recent development in connected vehicle technology (CVT), formally known as IntelliDrive or vehicle infrastructure integration (VII) in transportation community and as ad hoc networks (VANET) in wireless network community, has stimulated tremendous interests among decision makers, practitioners, and researchers due to the potential safety and mobility benefits provided by these technologies. Supported by the dedicated short range communication (DSRC) standard, connected vehicle technology will enable road vehicles to communicate with each other as well as to roadside infrastructure in the future; see an illustration in Figure 1. Thus, highways and streets will become an environment that encompasses ubiquitous computing and communication. Consequently, a new class of applications can be developed to dramatically increase safety, throughput, and energy efficiency. For example, CVT may serve as an ever-vigilant copilot to watch for potential hazards such as abrupt braking by a leading vehicle, a side vehicle in blind spot, and even a collision from behind. In addition, CVT supports various functionalities to relieve congestion such as notifying downstream congestion, alternative routes, and even parking information.
Moreover, CVT opens the door of enroute entertainment such as downloading music and video, checking e-mails, and maintaining social connections like Facebook. All of these possibilities depend on large-scale deployment of connected vehicle technology. However, a deployment decision has to take many factors into consideration. Among others, primary factors are the infrastructure needed for success and the degree of market penetration (i.e., percent of vehicles equipped with connected vehicle technology) required for effectiveness.

The above question is very difficult to answer because of the following: field experiments require a large-scale connected vehicle technology testbed which has yet to be deployed; simulation is unavailable since existing traffic simulation packages are not designed to model traffic enabled by connected vehicle technology; analytical modeling is prohibitive because of the complexity and interdependency involved in connected vehicle systems. To bypass these difficulties, this paper carries a humble goal by following a simplified modeling approach which is complemented by Monte Carlo simulation. In addition, the focus is to explore a feasible approach to conduct preliminary estimation of the mobility 


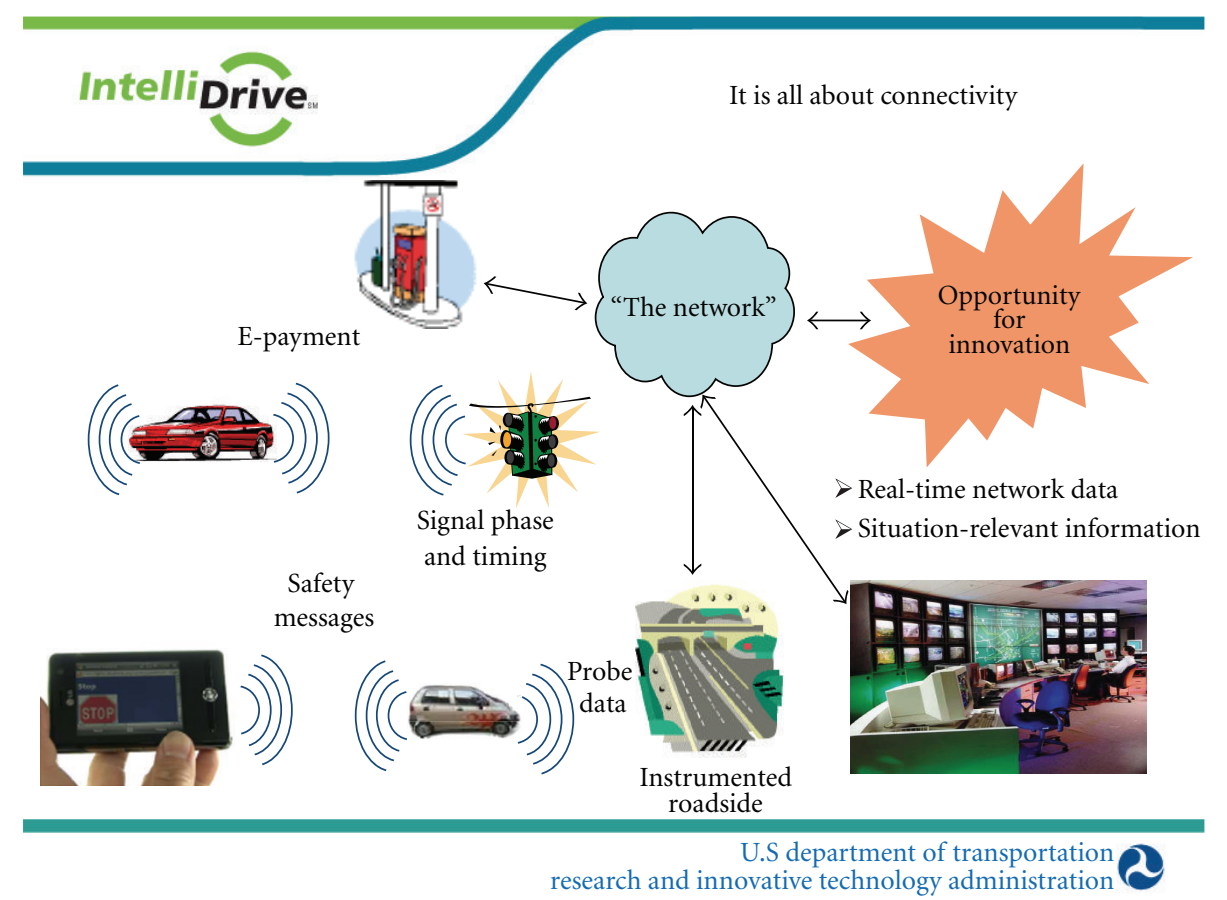

FIGURE 1: Connected vehicle technology.

benefit of connected vehicle technology, that is, the increase of highway capacity brought about by connected vehicle technology and how the result changes as market penetration varies. There are two building blocks in this approach. The first is to incorporate the effects of connected vehicle technology into driving behavior modeling. For such a purpose, a car-following model was derived based on the classical Gipps' model [1] by attributing the effects of connected vehicle technology to the change in the distribution of drivers' perception-reaction time. Recognizing that connected vehicle technology may bring other profound changes in traffic operations than merely perception-reaction time, the proposed model has to be kept as tractable as possible to make the analysis feasible yet capturing the major effect of connected vehicle technology. For this reason, some aspects of traffic flow, such as lane changing and hysteresis, are not modeled. Based on the proposed model, the second building block is a probabilistic analysis to provide an estimate of highway capacity. In this part, the major tools utilized are Wald's formula in probability theory and a theorem regarding the product moment of stopping time. An analytical approximate formula for capacity is obtained therein. A Monte Carlo simulation study is conducted to provide an alternative to verify this estimate since field tests are not possible at this time. The result obtained in this paper provides decision makers and practitioners with a basic tool to understand mobility benefit resulted from connected vehicle technology and how such a benefit varies as connected vehicle technology market penetration increases. In addition, using the methodology proposed in this paper, researchers can fine tune the assumption about the effects of connected vehicle technology to further investigate its benefits.
The remainder of this paper is organized as follows. In Section 2, relevant studies on this subject are briefly reviewed to provide a context in which the current paper fits. Next, in Section 3, the effects of connected vehicle technology are incorporated into the modeling of driving behavior by rectifying the Gipps' model. Following that, Section 4 is the probabilistic analysis and simulation verification. Section 5 provides an illustrative example to show the application of the methodology. Finally, the findings and results are summarized at the end.

\section{Existing Studies}

The idea of studying traffic flow benefits due to advanced technologies such as adaptive cruise control (ACC) systems and automated highway systems (AHS) has been addressed in the past. A great deal of studies have been identified which provided insights into highway capacity and traffic stability. A good survey of these studies can be found in [2]. A few additional references that present the necessary context for this study are added here. In their early studies on flow benefits of AHS, authors in [3,4] investigated how ACC affected traffic flow and found that the improvement in capacity is small. Also focused on ACC, authors in [5] studied the impact of ACC on traffic flow stability and found that car following based on a constant time headway is essentially unstable.

While traffic operation in a separate lane hosting only ACC vehicles represents an ideal condition, analyzing mixed traffic flow consisting of ACC-automated vehicles and manually operated vehicles poses more challenges. Reference [6] 
presented such a study. Their simulation results related the capacity trend to mixed ratios of ACC-equipped cars and their market penetration. They found that the capacity benefit became significant when ACC-equipped cars exceeded $50 \%$ market penetration. When all cars were equipped with the technology, they found a $33 \%$ increase in capacity.

In addition to considering mixed traffic, incorporating inter vehicular communication such as cooperative ACC (CACC) represents a more realistic scenario. Refernce [7] used Monte Carlo simulation to estimate lane capacity under varying proportions of autonomous ACC (AACC) and CACC. They concluded that AACC could only have a small impact on highway capacity (at most a $7 \%$ increase), while significant capacity gain could be expected with increased CACC market penetration (potentially more than doubling the capacity). Authors in [8] studied similar subject matter with a focus on the impacts of CACC on a highwaymerging scenario. Based on the traffic flow simulation model MIXIC, they found improved traffic stability and a slightly increased capacity compared to the non-AAC-equipped scenario.

In European Union, a simulation study was conducted on cooperative systems deployment impact assessment (CODIA). This study reported reduced average speed and hence increased journey times due to vehicle-infrastructure cooperation, and such an increase exhibited a quadratic "line of best fit" as market penetration varies from 0 to 100 percent [9].

Inspired by these original studies, our work considers a more general scenario which incorporates three types of driving modes enabled by connected vehicle technology (denoted as CVT thereafter), namely non-CVT, CVT assisted, and CVT automated. In the non-CVT mode, drivers operate their vehicles without any assistance from connected vehicle technology, just as what a regular driver does. In the CVTassisted mode, drivers receive connected vehicle technology assistances such as driver advisories (e.g., downstream congestion) and safety warnings (e.g., emergency brake), but these drivers still assume full control of their vehicles. The CVT-automated mode means that a vehicle is operated by CVT-enabled automatic driving features; however, the driver may break the loop and take over at any time as the need arises. In relation to these modes, existing studies emphasized the CVT-automated mode since ACC, AACC, and CACC can be considered as special cases of this mode. This paper broadens the perspective by also considering the effect due to CVT-enabled assistance to drivers (such as driver advisories and warnings). Moreover, this research takes a probabilistic approach and analytically relates the capacity benefit to the attributes of these driving modes and their varying market penetration rates. It is noted that CVT may result in increased throughput due to reduced accidents and suppressed congestion, and benefit of this nature is typically scenario dependent. As a generic approach, this paper explores the upper bound of such benefit, that is, the increased capacity, given that accidents and congestion have been prevented.

\section{Incorporating Connected Vehicle Technology Effects}

3.1. Assumptions and Simplifications. Connected vehicle technology can bring about many fundamental changes to transportation systems such as ubiquitous situational awareness, more efficient system control, more advanced safety features and. However, one thing remains the same: drivers will still have full control even though it may be delegated to CVT-enabled systems. Hence, it is reasonable to begin with driver modeling in order to predict the operations of CVT-enabled transportation systems. Among others, the major effects of connected vehicle technology on drivers are changes in the way that information is acquired, processed, and applied. For example, on-board radar can tell the subject driver exactly how far the leading and/or trailing vehicle is and how fast the gap closes, and wireless communication can warn the subject driver of an abrupt braking by the leading vehicle or the approaching of a fast vehicle behind. Given the mix of CVT-enabled and regular vehicles in the traffic, it is likely that rear ends might be resulted due to sudden and unexpected "automated" braking. Hopefully, CVT is able to monitor such hazard and warn the subject driver in advance. On-board computer can synthesize these sources of information and present the subject driver with driver advisories which allow CVT-assisted drivers to have a better understanding of their local and global contexts than drivers without such assistance. As such, assisted drivers may need less time to look out for information (e.g., accident ahead) and could plan accordingly in advance. Thus, they could focus more on understanding the information (e.g., expect emergency brake) and make control decisions. In addition, if a vehicle is so equipped, the information can also be processed before the result is delivered (e.g., a warning to slow down). These assistances may significantly reduce drivers' perception time, and they only need to concentrate on executing decisions which is related to reaction time. Moreover, the reaction time needed to execute decisions can be further shortened if a vehicle is running in the CVT-automated mode.

Therefore, central to driver modeling in CVT-enabled transportation systems is the modeling of driver perceptionreaction time. This modeling strategy is further supported by the following two considerations. First, the perceptionreaction time is a very, if not the most, significant parameter governing drivers' car-following behavior which directly affects traffic density and highway capacity. Such a parameter is very sensitive to stimuli from drivers' local context (such as in-vehicle assistance systems). This is also evident in various microscopic traffic models, in particular, the Gipps-type model [1] which follows a "safe-distance" argument. Other aspects of driving, such as vehicle handling, are intrinsic characteristics of drivers and less influenced by external information brought by connected vehicle technology. Secondly, connected vehicle technology provides real-time information to drivers. Though field experiments have yet to be conducted to verify this postulation, evidence in psychology literature such as in [10] indicates that perceptionreaction time strongly depends on the type and intensity of 
stimulus. Since CVT-enabled systems constitute a new type of stimulus with high intensity, such systems would shorten drivers' perception-reaction time, according to the literature.

Hence, it seems plausible to attribute the effects of connected vehicle technology to the distribution of drivers' perception-reaction time. For example, in the non-CVT mode, a driver typically needs to go through the full perception-reaction process and thus may necessitate a relatively long perception-reaction time (perhaps, a few seconds) on average. In addition, drivers without any assistance have less situational awareness which results in more uncertainty in their responses. This may give rise to a larger variance in their perception-reaction time. In the CVT-automated mode, the perception process is taken care of by connected vehicle technology, and the reaction process is handled by the automatic driving system. Thus, the resulting perception-reaction time can be minimal. Also, human drivers are not involved in the driving loop; therefore, the variance of perception-reaction time may be close to zero. In the CVT assisted mode, a wide range of possibilities may occur to the distribution. On one hand, it seems intuitive that CVT assistances such as advisories and warnings can greatly reduce drivers' perception time. On the other hand, such a new service may demand more attention to understand and familiarize and thus require a longer perception time, which is particularly true during confidence-building process. Before experimental data become available, the above discussion on perception-reaction time and their distributions remain open to debate. Nevertheless, it is reasonable to assume that the perception-reaction time of non-CVT, CVT-assisted, and CVTautomated drivers follow different distributions. Figure 2 is provided only for illustration purpose. Note that no assumption is made here regarding the actual distributions nor their relation. In particular, one should not take the figure as an implication that CVT-assisted and CVT-automated drivers have shorter perception-reaction times than nonCVT drivers. This treatment keeps the subsequent formulation generic and flexible for analysts to customize their models. For example, analysts can plug in suitable perception-reaction time distributions based on their own understanding or experiments in the field or on driving simulators. Remarkably, systems such INRETS MSIS [11], which integrated a driving simulator and a behavioral traffic simulation, can be a reasonable surrogate to field experiments in order to characterize the impacts of connected vehicle technology on drivers before its large-scale deployment. It is important to clarify at the beginning that the purpose of this paper is to formulate a methodology in generic terms to estimate the capacity benefit attainable from wide deployment of CVT, so that researchers, planners, and decision makers have a tool that can be tailored to their specific applications. It is recognized that the distributions of perception-reaction time under different driving modes are important inputs to the methodology. However, it is not the main focus of this paper to quantify these distributions which will be kept generic in subsequent discussion.

In addition, it is assumed that CVT-automated and CVTassisted modes are always able to reap the benefits of VANET, that is, such vehicles are always assumed to be in a vehicular

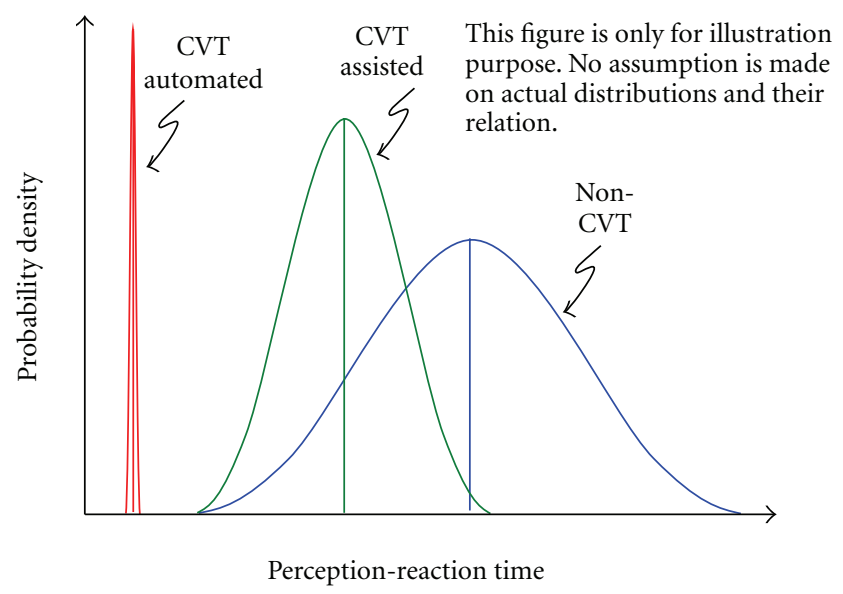

Figure 2: Perception-reaction time under different driving modes.

ad hoc network. It is recognized that such an assumption is not very true, especially under low CVT market penetration rates. Fortunately, this assumption is acceptable considering the following. First, it tends to overestimate highway capacity when there are not many CVT-equipped vehicles. Though not desirable, such an estimate does provide an upper bound of the capacity gained by connected vehicle technology. Second and perhaps more importantly, the validity of such an assumption increases when the deployment of connected vehicle technology is relatively significant, a scenario at which connected vehicle technology aims and under which connected vehicle technology makes the most sense. In order to fully account for this limitation, one must consider the dynamics of and interdependence between vehicular ad hoc networks and vehicular traffic. If this complication was to be taken into account, an analytical approach would no longer be adequate. Therefore, the goal of this research is to conduct a preliminary estimation of capacity benefit. Considering that field data are rare and the actual effects of connected vehicle technology are still subject to discussion, an easily understood and tractable approach seems more desirable to fulfill the purpose.

It is further assumed that CVT market penetration rate (i.e., the percent of total vehicles operating in each of the three modes) is known. With the above setup, it is straightforward to derive a car-following model with perceptionreaction time as a parameter. Compared with the original [1] model, the new model rectifies the perception-reaction time which considers CVT-enabled driving modes and incorporates their market penetration rates. This model is then used to derive an equilibrium flow-density relationship, from which maximum flow rate (i.e., the capacity) can be derived. Considering the random nature of the perception-reaction time, a probabilistic analysis is performed to investigate the properties of the capacity, and a Monte Carlo simulation is used to verify the results obtained above.

3.2. Model Formulation. Perception-reaction time plays a significant role in car following and traffic operations. Gipps' model [1] stipulates that, at any moment, a vehicle should 


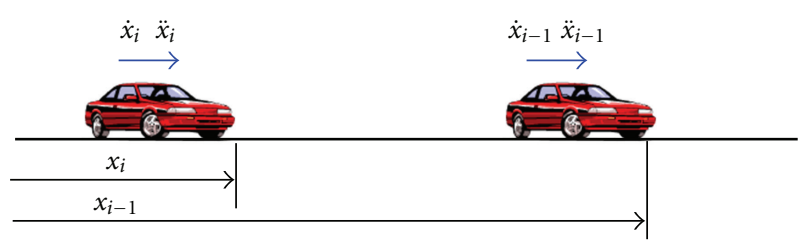

Figure 3: Vehicles in car following.

leave enough room in front of it in order to be able to stop safely behind its leading vehicle in the event that the leading vehicle applies emergency brake. Figure 3 shows two vehicles in car following. The leading vehicle with ID $i-1$ is at position $x_{i-1}$ with speed $\dot{x}_{i-1}$ and acceleration $\ddot{x}_{i-1}$. The subject vehicle $i$ is at position $x_{i}$ with speed $\dot{x}_{i}$ and acceleration $\ddot{x}_{i}$. The minimum safe distance should allow the subject vehicle to stop behind the leading vehicle after a perception-reaction time $\tau_{i}$ and a deceleration process at a comfortable level $b_{i}=\ddot{x}_{i}<0$. To be specific, the stopped position of the leading vehicle if it applies an emergency brake at time $t$ is

$$
x_{i-1}^{*}=x_{i-1}(t)-\frac{\dot{x}_{i-1}^{2}(t)}{2 B_{i-1}}
$$

where $B_{i-1}$ is the maximum deceleration $\left(\ddot{x}_{i-1}^{\max }<0\right)$ applied by the leading vehicle. The stopped position of the subject vehicle is

$$
x_{i}^{*}=x_{i}(t)+\dot{x}_{i} \tau_{i}-\frac{\dot{x}_{i}^{2}(t)}{2 b_{i}} .
$$

To ensure safety, the following relationship must hold:

$$
x_{i-1}^{*}-l_{i-1} \geq x_{i}^{*},
$$

where $l_{i-1}$ is the length of the leading vehicle. Putting the above together, the safe distance for the subject vehicle can be expressed as

$$
D_{i}(t)=x_{i-1}(t)-l_{i-1}-x_{i}(t) \geq \dot{x}_{i} \tau_{i}-\frac{\dot{x}_{i}^{2}(t)}{2 b_{i}}+\frac{\dot{x}_{i-1}^{2}(t)}{2 B_{i-1}},
$$

which corresponds to a spacing of

$$
S_{i}(t)=x_{i-1}(t)-x_{i}(t) \geq l_{i-1}+\dot{x}_{i} \tau_{i}-\frac{\dot{x}_{i}^{2}(t)}{2 b_{i}}+\frac{\dot{x}_{i-1}^{2}(t)}{2 B_{i-1}} \text {. }
$$

Under equilibrium conditions, vehicles tend to behave uniformly and thus lose their identities. After suppressing time $t$, the spacing becomes

$$
S \geq l+\dot{x} \tau-\frac{\dot{x}^{2}}{2 b}+\frac{\dot{x}^{2}}{2 B}=\left(\frac{1}{2 B}-\frac{1}{2 b}\right) \dot{x}^{2}+\tau \dot{x}+l .
$$

Following Gipps' argument, an additional delay $\theta$ to $\tau$ offers extra protection for the subject vehicle, so the above inequality is turned into

$$
S=l+\dot{x}(\tau+\theta)-\frac{\dot{x}^{2}}{2 b}+\frac{\dot{x}^{2}}{2 B}=\left(\frac{1}{2 B}-\frac{1}{2 b}\right) \dot{x}^{2}+(\tau+\theta) \dot{x}+l
$$

Thus, the safe car-following distance, or equivalently the safe spacing, is explicitly expressed as a function of speed $v \equiv \dot{x}$ (under equilibrium conditions, it is also the traffic speed) with parameters $\tau, \theta, B, b$, and $l$. Among all the parameters, $\tau$ and $\theta$ characterize the behavior of drivers and are independent of speed $v$ and spacing $S \cdot B, b$, and $l$ are vehicle properties and can be assumed as constants. Since we also know that density $k$ is related to spacing $S$,

$$
k=\frac{1}{S}
$$

the flow $q$ is obtained by substituting $k$ and $v$ into the fundamental relation

$$
q=k v=\frac{v}{G v^{2}+\tau^{\prime} v+l},
$$

where $\tau^{\prime}=\tau+\theta$ and $G=(1 / 2 B)-(1 / 2 b)$. In this relation, $v$ can be viewed as the primary input. $v$ and $\tau^{\prime}$ are independent variables. The maximum attainable $q$ is of interest. To find the maximum $q$ (denoted $q_{m}$ ), we solve the equation

$$
\left.\frac{d q}{d v}\right|_{v_{m}}=-\left.\frac{G-\left(l / v^{2}\right)}{\left(G v+\tau^{\prime}+(l / v)\right)^{2}}\right|_{v_{m}}=0
$$

We get the root

$$
v_{m}=\sqrt{\frac{l}{G}}
$$

and correspondingly,

$$
q_{m}=\frac{1}{2 \sqrt{G l}+\tau^{\prime}} .
$$

To verify that $q_{m}$ is indeed a maximum as $v$ varies, one may simply check the second derivative of $q$ at $v_{m}$. It turns out that this is true.

\section{Probabilistic Analysis}

4.1. The Stopping Time Formulation with Random $\tau$. Note that the above discussion does not incorporate the random nature of perception-reaction time $\tau$ nor its distributions under different driving modes. To begin with, the above equation allows the calculation of capacity $q_{m}$ given perception-reaction time $\tau$ if it is uniform across the entire driver population. Such a case is simple but unrealistic. A step forward would be to assume uniform perceptionreaction time under each driving mode. Combined with market penetration (analogous to the probability of each driving mode), these perception-reaction times can be used to estimate the average (i.e., mathematical expectation) capacity as the result of the entire driver population. This subsection deals with a more realistic scenario which assumes different distributions of perception-reaction time under different driving modes, and the estimation of capacity is to compute the mathematical expectation of $q_{m}$ based on these underlying distributions and their market penetration rates.

Denote $f_{\text {no }}(t)$ the probability density of perceptionreaction time of drivers under the non-CVT with mean $\tau_{\text {no }}$ 
and variance $\operatorname{Var}\left(\tau_{\text {no }}\right)$. Similarly, the probability density of the CVT-assisted mode is $f_{\text {as }}(t)$ with mean $\tau_{\text {as }}$ and variance $\operatorname{Var}\left(\tau_{\mathrm{as}}\right)$; the probability density of the CVT-automated mode is $f_{\mathrm{au}}(t)$ with mean $\tau_{\mathrm{au}}$ and variance $\operatorname{Var}\left(\tau_{\mathrm{au}}\right)$. In addition, market penetration rates of road vehicles operating in nonCVT, CVT-assisted, and CVT-automated modes are denoted as $p_{\text {no }}, p_{\text {as }}$, and $p_{\text {au }}$ respectively. They satisfy the following relationships: $0 \leq p_{\text {no }}, p_{\mathrm{as}}, p_{\mathrm{au}} \leq 1$, and $p_{\mathrm{no}}+p_{\mathrm{as}}+p_{\mathrm{au}}=$ 1. Therefore, the perception-reaction time of an individual driver $i, \tau_{i}$, is a random variable which can be modeled by drawing first from the percent/probability of market penetration to determine which driving mode this driver uses and then from the distribution of perception-reaction time of that particular mode.

Henceforth, we will investigate the properties of $q$ and $q_{m}$ as $\tau$ takes on random values. Usually, the first-order second moment analysis (FOSM) is sufficient to fulfill this purpose. However, since FOSM is based on the Taylor expansion of functions, the accuracy of approximation relies heavily on the convergence rate of the Taylor series in the neighborhood of the expansion. For the higher-order moment, this is especially true. In this situation, it is unfortunate that the expression of $q_{m}$ is ill posed to adopt the FOSM. This is because $q_{m}$, written in the form of $f(x)=1 /(a+b x)$, corresponds to a slowly converging expansion series when $|a+b x| \sim 0$, a result of comparable values of $a$ and $b$.

Thus, we tackle the problem in a different way. In particular, we introduce the stopping time concept such that the expansion-based analysis like FOSM is avoided. The procedure is as follows. First, we redefine the flow as

$$
q=k v=\frac{N}{L} v
$$

where $v$ is the traffic speed, $L$ is the length of a segment of highway in consideration, and $N$ is the number of vehicles within the length. Flow $q$ can be written as $N /(L / v)$ and interpreted as the number of vehicles occupying a certain length of road divided by the time they take to traverse the road. Under equilibrium conditions, this definition is equivalent to the original definition. Then we can adopt the concept of random walk. It is easy to see that $N$ is actually the stopping time (stopping time, a standard concept in probability theory, can be roughly regarded as a "random time" whose value depends on current and historical values of a stochastic process). A rigorous definition is found in [12] where a random walk $\sum_{i=1}^{n} S_{i}$ with positive drift $E\left(S_{i}\right)$ has

$$
N=\inf _{n}\left\{n: \sum_{i=1}^{n} S_{i}>L\right\},
$$

where inf indicates the infinium of a set,

$$
S_{i}=G v^{2}+\tau^{\prime} v+l
$$

then

$$
\mu_{q} \equiv E(q)=\frac{v}{L} E(N)
$$

Moreover,

$$
E(N)=\frac{L}{E\left(S_{i}\right)}=\frac{L}{G v^{2}+\mu_{\tau^{\prime}} v+l},
$$

where the first equality is due to Wald's equation, with its form and derivation given in [12]. Application of this equation requires $E\left(S_{i}\right)<\infty$, which is obviously true from a realistic point of view. Thus, we obtain the approximation of expected capacity when speed is $v$,

$$
\mu_{q} \equiv E(q) \sim \frac{v}{G v^{2}+\mu_{\tau^{\prime}} v+l},
$$

where $\mu_{\tau^{\prime}}=E\left(\tau^{\prime}\right)$. Plugging in the optimal speed $v=v_{m}=$ $\sqrt{l / G}$, we obtain the maximum value of approximation of expected capacity,

$$
\mu_{q, m} \equiv E\left(q_{m}\right) \sim \frac{1}{2 \sqrt{G l}+\mu_{\tau^{\prime}}} .
$$

To obtain the variance of $q$ and $q_{m}$, we need to utilize a formula regarding the variance of stopping time given in [13]. In the current scenario, it is

$$
\operatorname{Var}(N)=\mu^{-3} \sigma^{2} L+\mu^{-2} K+o(1)
$$

where $K$ is a constant independent of $L$,

$$
\begin{gathered}
\mu=E\left(S_{i}\right)=G v^{2}+\mu_{\tau^{\prime}} v+l, \\
\sigma^{2}=\operatorname{Var}\left(S_{i}\right)=\sigma_{\tau^{\prime}}^{2} v^{2} .
\end{gathered}
$$

By substituting them into the definition of $q$, by letting $L$ be large enough, and by only keeping the dominating term, we get the variance of flow in the general case

$$
\sigma_{q}^{2} \equiv \operatorname{Var}(q) \sim \frac{\sigma_{\tau^{\prime}}^{2} v^{4}}{\left(G v^{2}+\mu_{\tau^{\prime}} v+l\right)^{3} L}
$$

Plugging in the optimal speed $v=v_{m}=\sqrt{l / G}$, we obtain the approximate variance of the maximum flow, that is, capacity,

$$
\begin{aligned}
\sigma_{q, m}^{2} \equiv \operatorname{Var}\left(q_{m}\right) & \sim \frac{\sigma_{\tau^{\prime}}^{2} l^{2}}{\left(2 l+\mu_{\tau^{\prime}} \sqrt{l / G}\right)^{3} G^{2} L} \\
& =\frac{\sigma_{\tau^{\prime}}^{2} G l}{\left(2 G l+\mu_{\tau^{\prime}} \sqrt{G l}\right)^{3}} \frac{l}{L}
\end{aligned}
$$

We see that two quantities, $\sqrt{G l}$ and $l / L$, together with characteristics of perception-reaction time $\tau^{\prime}$ determine the variance. It is notable that the involved quantities are all easily measured, indicating the advantage of our estimate formula in terms of calibration.

4.2. Simulation Verification. To verify the creditability of the above approximation formulas, it is necessary to device an independent approach, preferably simulation since largescale field experiments are infeasible. Considering that a traffic simulation package that is capable of capturing the effects of connected vehicle technology is not available, this study employs a Monte Carlo simulation which repeatedly samples a traffic system by drawing from underlying distributions. 


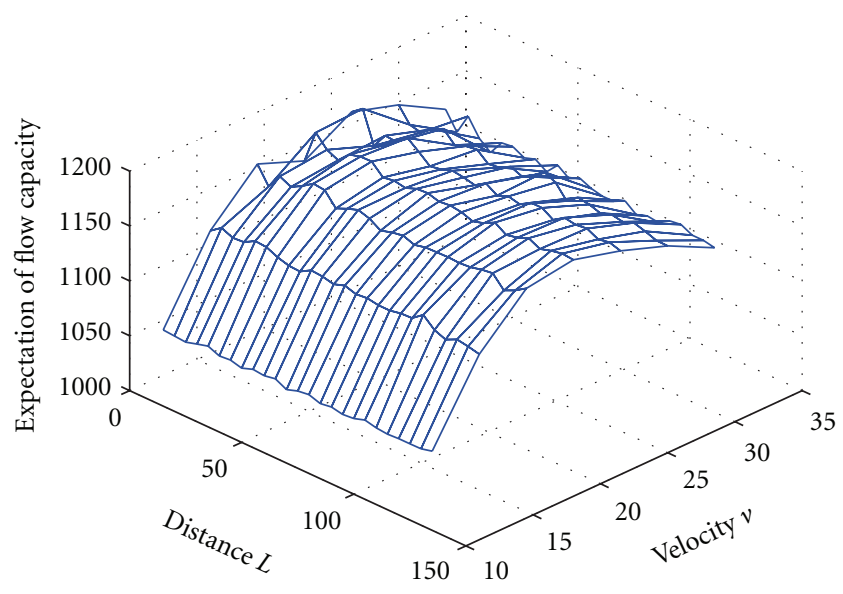

(a)

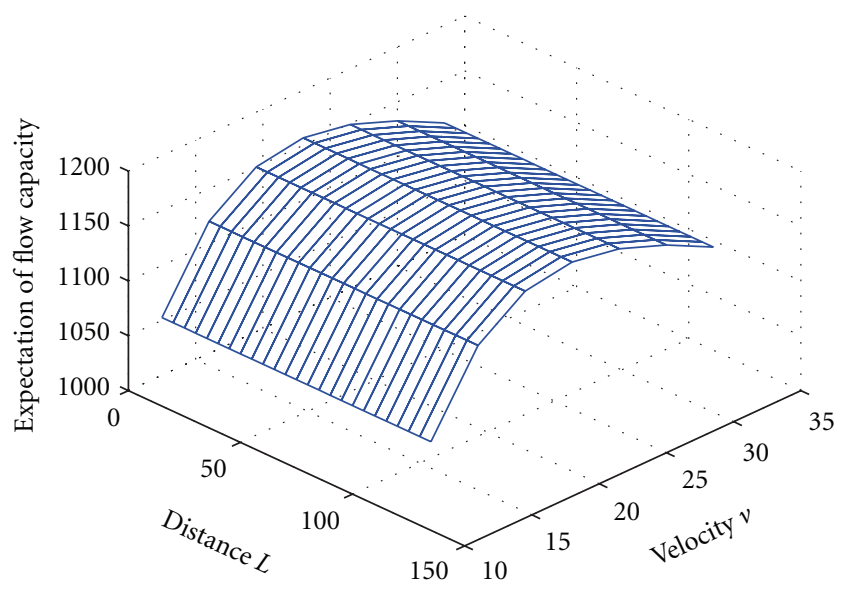

(b)

FIGURE 4: The comparison of simulated and approximate expectation of flow $\mu_{q}$ (in passenger cars per hour per lane or pcphpl) ((a) simulated value; (b) approximate value).

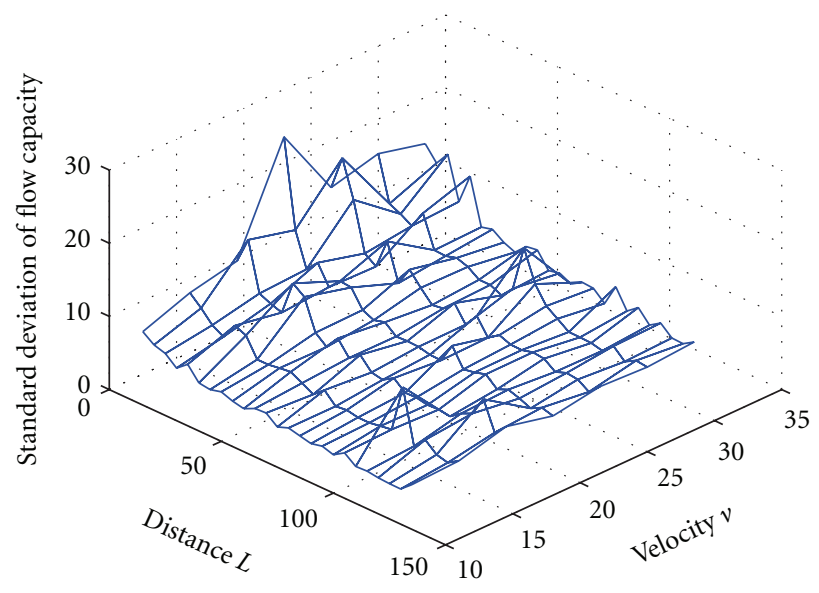

(a)

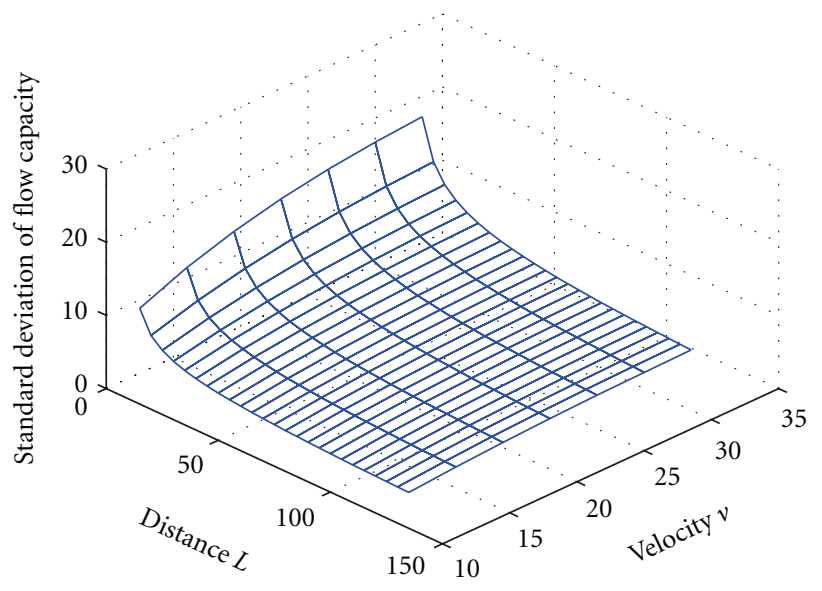

(b)

FIGURE 5: The comparison of simulated and approximate standard deviation of flow $\sigma_{q}$ (in pcphpl) ((a) simulated value; (b) approximate value).

The number of trials depends on the precision required with one trial as follows:

(1) select road length in consideration, denoted $L$, and initialize $L_{0}=0$,

(2) randomly sample the perception-reaction time $\tau$ and calculate the cumulative length, which is defined as $L_{j+1}=L_{j}+S$,

(3) if $L_{j}>L$, denote $N=\max j$, calculate $q=v N / L$, and else return to (2).

Each trial in the simulation mimics the instantaneous vehicle spatial distribution on the road, such that the count of vehicles $N$ at each moment is obtained. In the simulation, we assume that the random perception-reaction time has a density of the following form:

$$
f_{\tau}(t)=p_{\mathrm{au}} f_{\mathrm{au}}(t)+p_{\mathrm{as}} f_{\mathrm{as}}(t)+p_{\mathrm{no}} f_{\mathrm{no}}(t)
$$

where $\sum_{i} p_{i}=1$ and $i \in\{\mathrm{au}$, as, no $\}$. Here, $f_{i}(\cdot)$ 's represents the density of the perception-reaction time distribution of the $i$ th group, and $p_{i}$ 's are interpreted as the market penetration of the corresponding groups. For the purpose of illustration, we consider an ideal and simplified case. We assume that the $f_{i}$ 's are the density of uniform random variables. The function $f_{i}$ is of the form

$$
f_{i}(t)=\frac{1}{u_{i}-l_{i}} I\left(t \in\left[l_{i}, u_{i}\right]\right) .
$$

It is easy to see that a random variable with the above density $f_{i}$ has an expectation $\mu_{i}=\left(u_{i}+l_{i}\right) / 2$ and a variance $\sigma_{i}=$ $\left(u_{i}-l_{i}\right)^{2} / 12$. We then have

$$
\mu_{\tau}=\sum_{i=1}^{3} p_{i} \mu_{i}
$$

$$
\sigma_{\tau}=\sqrt{\sum_{i=1}^{3} p_{i} \mu_{i, 2}-\mu_{T}^{2}}=\sqrt{\sum_{i=1}^{3} p_{i}\left(\sigma_{i}^{2}+\mu_{i}^{2}\right)-\mu_{T}^{2}}
$$




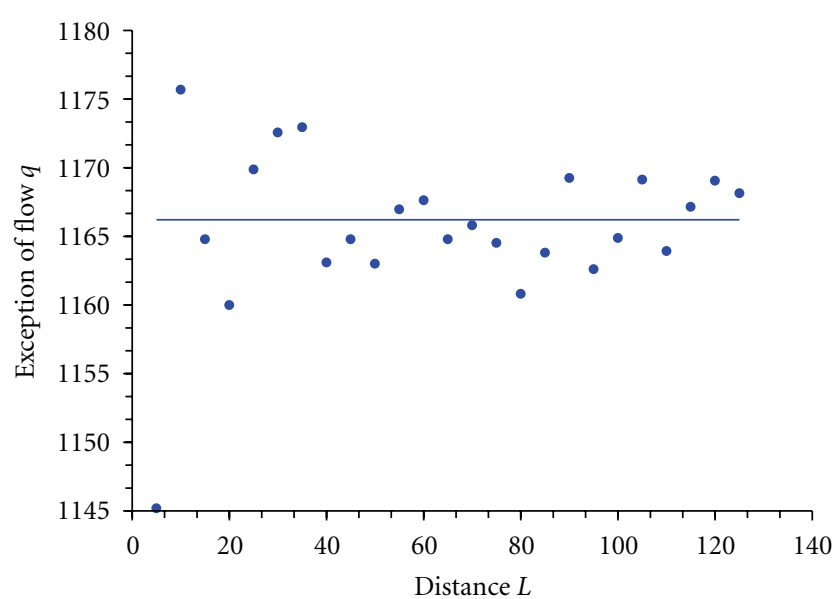

FIgURE 6: The comparison of simulated and approximate expectation of maximum flow $\mu_{q, m}$ (distance $L, m$; flow $q$, pcphpl; dots: simulated values; solid line: approximate values).

Let the first, second, and third terms in expression of $f_{T}$ represent the CVT-automated, CVT-assisted, and non-CVT groups, respectively, then we assume that

$$
\begin{aligned}
\mu_{\mathrm{au}}=0.5, & \mu_{\mathrm{as}}=1.0, & \mu_{\mathrm{no}}=1.5, \\
\sigma_{\mathrm{au}}=0, & \sigma_{\mathrm{as}}=0.2, & \sigma_{\mathrm{no}}=0.5, \\
p_{\mathrm{au}}=0.2, & p_{\mathrm{as}}=0.5, & p_{\mathrm{no}}=0.3 .
\end{aligned}
$$

Moreover, we fix $B=-4 \mathrm{~m} / \mathrm{sec}^{2}, b=-2 \mathrm{~m} / \mathrm{sec}^{2}, l=$ $4.5 \mathrm{~m}$ ( 15 feet), and $\tau^{\prime}=1.5 \tau$ (then there is $\mu_{\tau^{\prime}}=1.5 \mu_{\tau}$ and $\sigma_{\tau^{\prime}}=1.5 \sigma_{\tau}$ ). The number of iterations in each loop is 1000 . The lengths of the segments on the one-lane highway vary from $5 \mathrm{~km}$ to $125 \mathrm{~km}$, with a step of $5 \mathrm{~km}$. The traffic speed varies from $v_{\mathrm{opt}}-10.5 \mathrm{~km} / \mathrm{hr}$ to $v_{\mathrm{opt}}+10.5 \mathrm{~km} / \mathrm{hr}$, with a step of $3.5 \mathrm{~km} / \mathrm{hr}$. The $v_{m}$ is the optimal speed defined above.

Observation of the simulation results and the conclusions drawn are as follows. First, in the case under consideration, the approximate expectation $\mu_{q}$ and standard deviation $\sigma_{q}$ of the flow are close to the simulation results, as shown in Figures 4 and 5. Second, in particular, the comparison of $\mu_{q, m}$ and $\sigma_{q, m}$ with the simulation is shown in Figures 6 and 7. The relative error of $\mu_{q, m}$ is very small, around $1 \%$. For the standard deviation $\sigma_{q, m}$, we see the fit of approximation to simulation is also near perfect, especially as the distance $L$ gets larger. To summarize, the simulation of the case when $\tau$ takes a specific distribution numerically justifies the approximations obtained by probabilistic analysis, and it intuitively illustrates the quality of these approximations.

\section{An Illustrative Example}

The simulation study in the above section is able to provide an estimate of the capacity gain in absolute terms. Such a result, however, is lower than the typical capacity under ideal conditions, that is, 2400 pcphpl for a basic freeway section. This is due to the conservative nature of the Gipps model, which is less capable of capturing the close-following

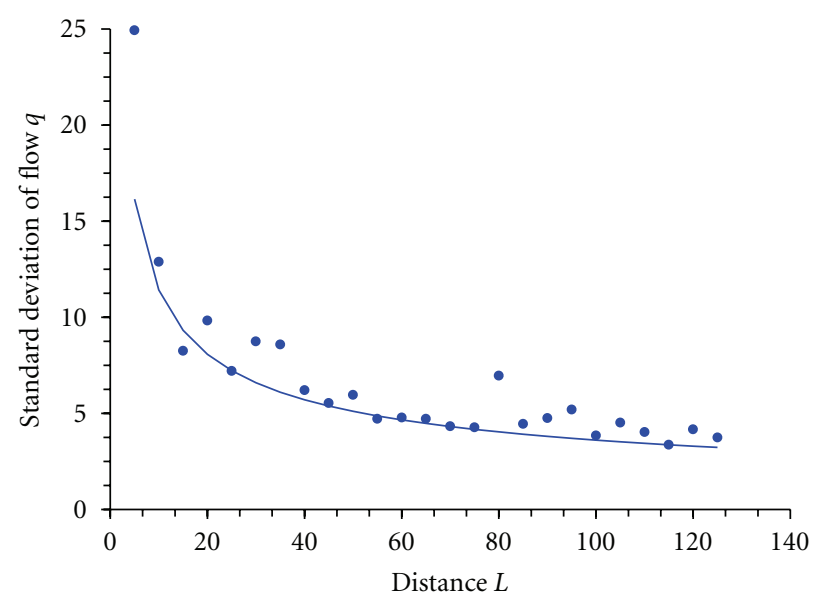

FIgURE 7: The comparison of simulated and approximate standard deviation of maximum flow $\sigma_{q, m}$ (distance $L, m$; flow $q$, pcphpl; dots: simulated values; solid line: approximate values).

behavior in reality. Developing a more realistic model may resolve the problem, but the mathematical tractability may be lost as well. Therefore, it is reasonable to describe the capacity benefit in relative terms, as discussed below.

To answer the question at the beginning of this paper (i.e., degree of market penetration required for effectiveness), we provide the following illustrative example. This example consists of four cases, and in each case, the ratio $p_{a u} / p_{a s}$ is assumed to be constant. In addition, we define the relative change in capacity as

$$
\begin{aligned}
r\left(\frac{p_{\mathrm{au}}}{p_{\mathrm{as}}}, p_{\mathrm{no}}\right)= & \frac{q_{m}\left(p_{\mathrm{au}}, p_{\mathrm{as}}, p_{\mathrm{no}}\right)}{q_{m}(0,0,1)} \\
= & \frac{q_{m}\left(\left(1-p_{\mathrm{no}}\right)\left(\left(p_{\mathrm{au}} / p_{\mathrm{as}}\right) /\left(p_{\mathrm{au}} / p_{\mathrm{as}}+1\right)\right)\right.}{q_{m}(0,0,1)}, \\
& \frac{\left.\left(1-p_{\mathrm{no}}\right)\left(1 /\left(p_{\mathrm{au}} / p_{\mathrm{as}}+1\right)\right), p_{\mathrm{no}}\right)}{q_{m}(0,0,1)},
\end{aligned}
$$

where $q_{m}(\cdot, \cdot, \cdot)$ is the capacity corresponding to market penetration $\left(p_{\text {au }}, p_{\text {as }}, p_{\text {no }}\right)$, and the second equality is $p_{\text {au }}+$ $p_{\text {as }}+p_{\text {no }}=1$. This formula could be interpreted as the ratio of increased capacity over the original capacity (i.e., $\left.p_{\mathrm{no}}=100 \%\right)$. By employing this definition, we will hopefully overcome the lower estimate by the Gipps' model. We obtain the values of $r$ in four cases, that is, when $p_{\mathrm{au}} / p_{\mathrm{as}}=$ $0.1,1,10,100$. The results are as shown in Figure 8. It is found that the increase of capacity ranges between $20 \%$ and $50 \%$ when connected vehicle technology is fully deployed (i.e., $p_{\text {no }}=0$ ), with the former case corresponding to $p_{\mathrm{au}} / p_{\mathrm{as}}=0.1$ and the latter case $p_{\mathrm{au}} / p_{\mathrm{as}}=100$. The result seems to suggest that the change of the $p_{\text {au }} / p_{\text {as }}$ ratio from 1 to 10 has much stronger effect than that from 10 to 100 . A plausible interpretation is that, with high percentage of CVT-assisted vehicles in the traffic, drivers have more chances to negotiate and hence more room of improvement. As the traffic is dominated by CVT-automated vehicles, they 

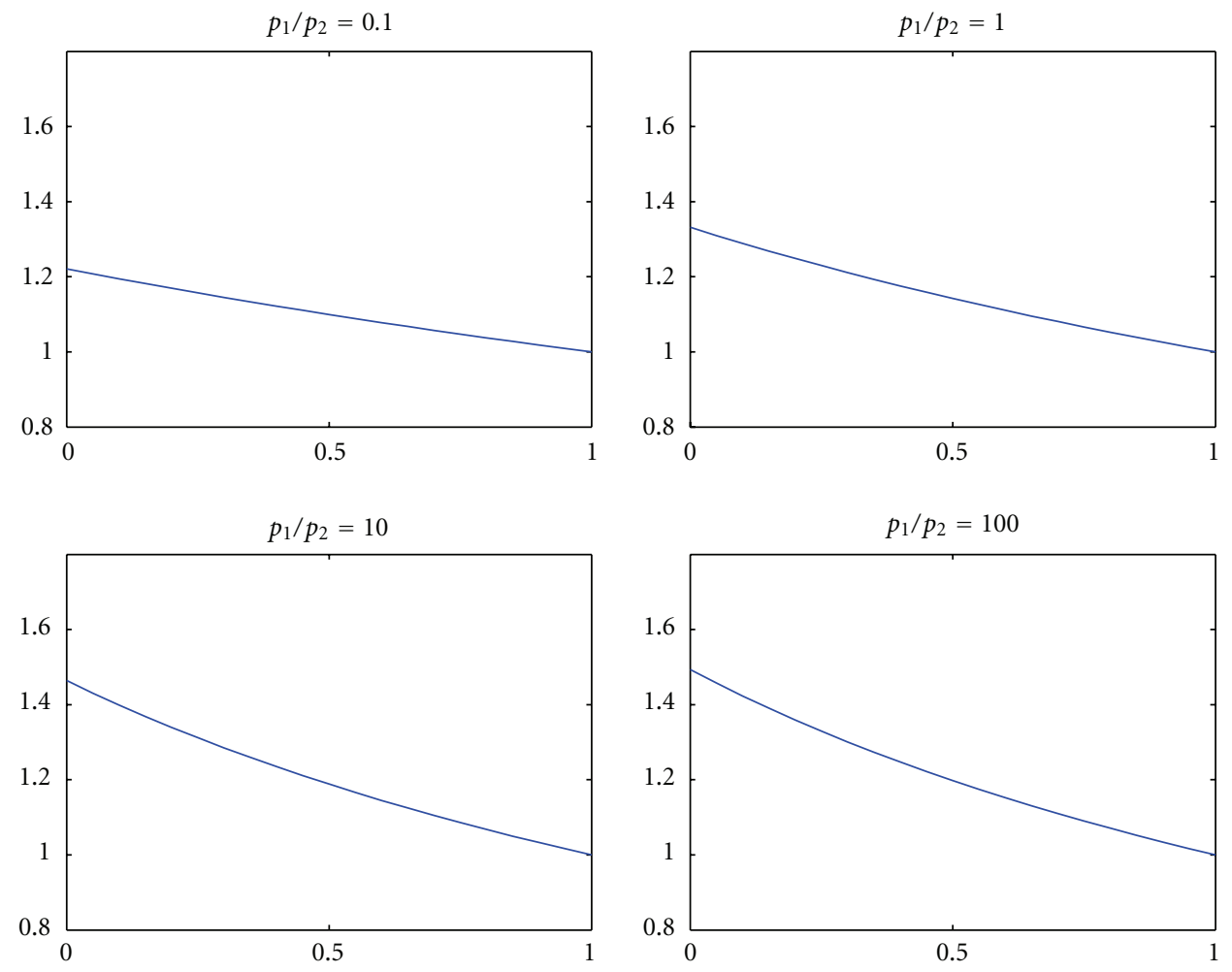

FIGURE 8: The relative benefits of CVT as function of market penetration of CVT in different cases ( $x$-axis: market penetration of nonassisted vehicles; $y$-axis: ratio of increased capacity to the original capacity).

move like a train whose already optimized performance allows little room for improvement. Note that the above example is only a rough estimate under some assumptions and simplifications. Nevertheless, the example does indicate that the benefit from employing connected vehicle technology could be quite significant even when the market penetration of CVT-automated vehicles is small (given full CVT deployment). As more accurate information regarding the involved parameters becomes available, the estimate can be finetuned, and more accurate results are expected. The outcomes can be used to help make the decision on connected vehicle technology deployment in future.

\section{Concluding Remarks}

The purpose of this study was to provide a methodology to estimate the capacity benefit obtained from CVT deployment. To fulfill this purpose, the classical Gipps' model was modified to incorporate the effects of CVT, and a probabilistic approach was presented to analyze the impact of such effects on highway capacity. In particular, approximate formulas of expectation and variance of the capacity were derived in a random setting. A simulation study was conducted to numerically verify the creditability of these formulas. Also included was an illustrative example that applied the proposed methodology. Under some assumptions and simplifications, the example showed that the increase of capacity ranges between $20 \%$ and $50 \%$ when CVT is fully deployed. Note that the lower bound, which corresponds to a relatively small market penetration of CVT-automated vehicles, may still yield quite significant capacity gain. Findings of this study, in general, agree with previous studies [6-8] on related subject, but this paper provides more indepth information such as the curve of capacity gain as market penetration changes and the methodology to estimate such curve.

Though the proposed methodology was motivated by capturing the effects of CVT (i.e., its impacts on three driving modes), such a methodology is readily applicable to a traffic system involving multiple classes of drivers (e.g., teenagers, middle-aged, and senior drivers whose perception-reaction times may follow different distributions). Meanwhile, the study may be further extended to more complicated and realistic scenarios (e.g., nonequilibrium flow and nonhomogeneous types of vehicles) where more involved simulation is expected before field experiments in a large-scale testbed become feasible. Since the distributions of perceptionreaction time under different driving modes are critical to estimation results, further studies particularly those of human factors are necessary to quantify these distributions.

\section{Acknowledgments}

This research is partly supported by U.S. Department of Transportation University Transportation Center Program. The authors would like to thank the anonymous reviewers for their valuable comments and suggestions to improve the quality of the paper. 


\section{References}

[1] P. G. Gipps, "A behavioural car-following model for computer simulation," Transportation Research Part B, vol. 15, no. 2, pp. 105-111, 1981.

[2] J. VanderWerf, S. Shladover, N. Kourjanskaia, M. Miller, and H. Krishnan, "Modeling effects of driver control assistance systems on traffic," Transportation Research Record, no. 1748, pp. 167-174, 2001.

[3] B. Rao and P. Varaiya, "Flow benefits of autonomous intelligent cruise control in mixed manual and automated traffic," Transportation Research Record, vol. 1408, pp. 36-43, 1993.

[4] B. Rao, P. Varaiya, and F. Eskafi, "Investigations into achievable capacities and stream stability with coordinated intelligent vehicles," Transportation Research Record, vol. 1408, pp. 27-35, 1993.

[5] S. Darbha and K. R. Rajagopal, "Intelligent cruise control systems and traffic flow stability," Transportation Research Part C, vol. 7, no. 6, pp. 329-352, 1999.

[6] T. H. Chang and I. S. Lai, "Analysis of characteristics of mixed traffic flow of autopilot vehicles and manual vehicles," Transportation Research Part C, vol. 5, no. 6, pp. 333-348, 1997.

[7] J. VanderWerf, S. Shladover, M. Miller, and N. Kourjanskaia, "Evaluation of the effects of adaptive cruise control systems on highway traffic flow capacity and implications for deployment of future automated systems," in Proceedings of the 81st TRB Annual Meeting, 2001, Pre-Print CD-ROM.

[8] B. van Arem, C. J. G. van Driel, and R. Visser, "The impact of cooperative adaptive cruise control on traffic-flow characteristics," IEEE Transactions on Intelligent Transportation Systems, vol. 7, no. 4, pp. 429-436, 2006.

[9] K. Kulmala, P. Levikangas, N. Sihvola et al., "Co-operative systems deployment impact assessment (CODIA) deliverable 5: final study report," Tech. Rep., VTT Technical Research Centre of Finland, 2008

[10] R. D. Luce, Response Times: Their Role in Inferring Elementary Mental Organization, Oxford University Press, New York, NY, USA, 1986.

[11] S. Espi and J.-M. Auberlet, "ARCHISIM: a behavioural multiactors traffic simulation model for the study of a traffic system including ITS aspects," International Journal of ITS Research, vol. 5, no. 1, pp. 7-16, 2007.

[12] R. Durrett, Probability: Theory and Examples, Duxbury Press, 1996.

[13] T. Lai and D. Siegmund, "A nonlinear renewal theory with applications to sequential analysis. II," Annuals of Statistics, vol. 7, no. 1, pp. 60-76, 1979. 

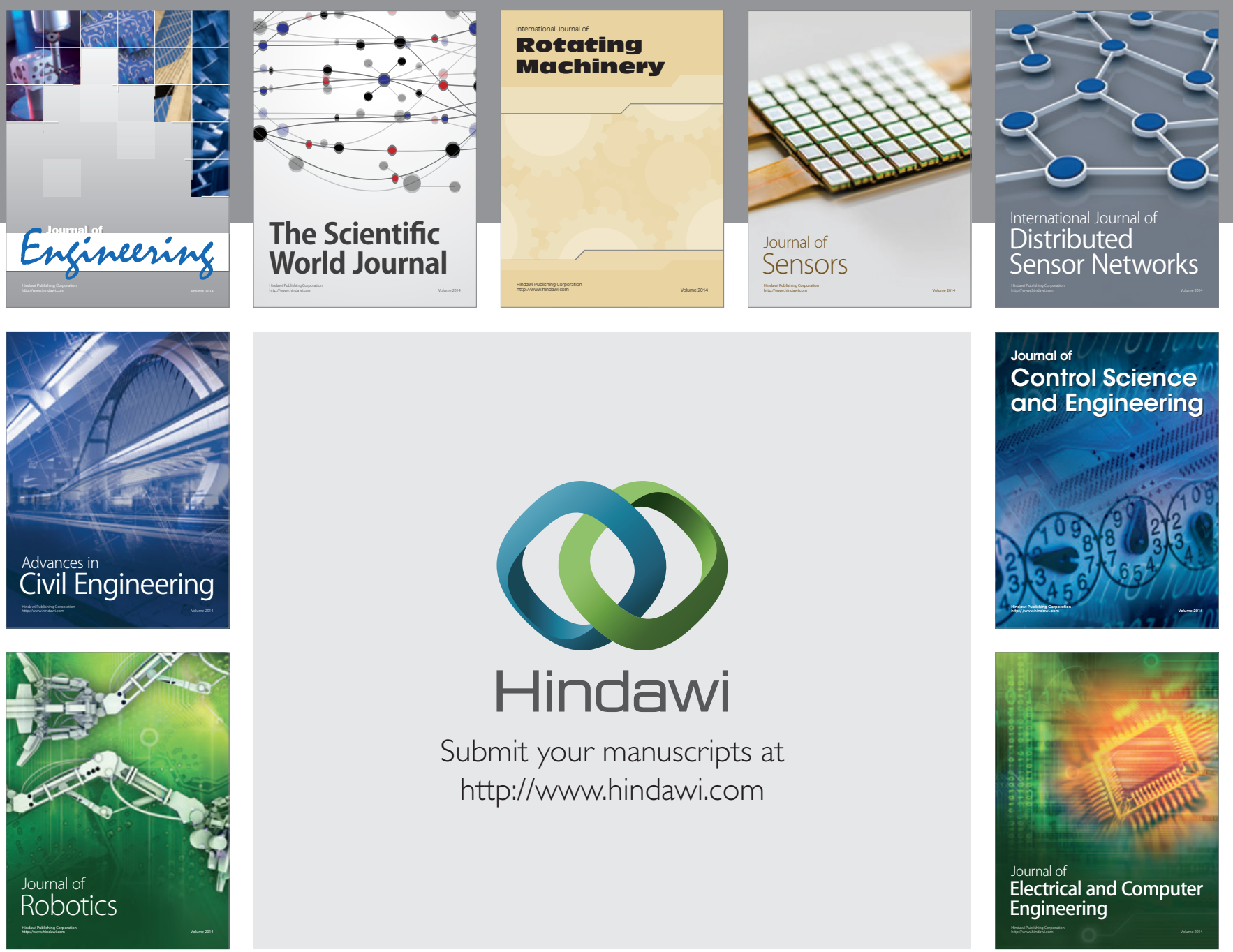

Submit your manuscripts at

http://www.hindawi.com
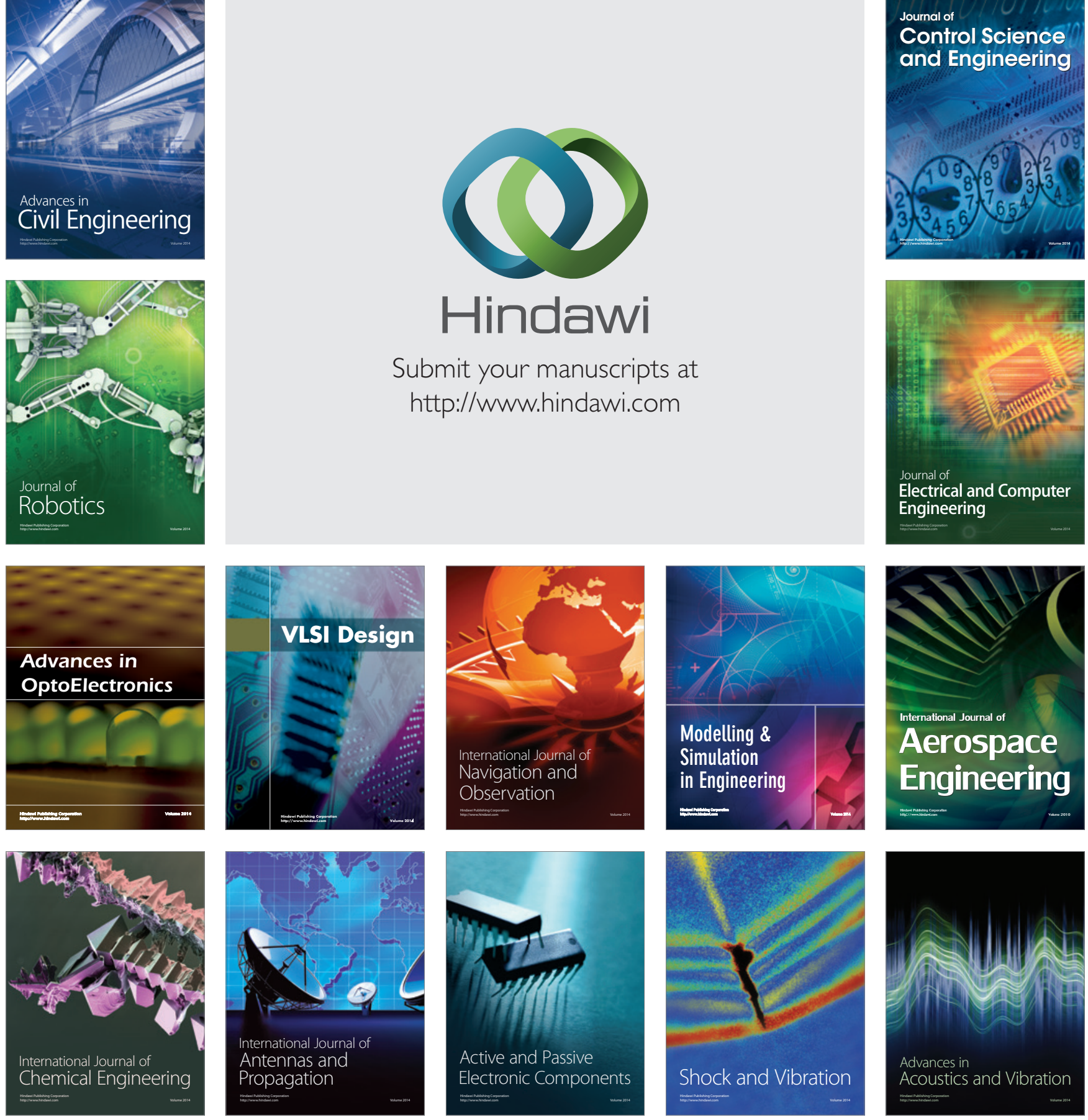